7. Beuche, F. Physical Properties of Polymers (Interscience, New York, 1962).

8. Smith, S. B., Finzi, L. \& Bustamante, C. Direct mechanical measurements of the elasticity of single DNA molecules by using magnetic beads. Science 258, 1122-1126 (1992).

9. Smith, S. B., Cui, Y. \& Bustamante, C. Overstretching B-DNA: the elastic response of individual double-stranded and single-stranded DNA molecules. Science 271, 795-799 (1996).

10. Wang, M. D., Yin, H., Landick, R., Gelles, J. \& Block, S. M. Stretching DNA with optical tweezers. Biophys. J. 72, 1335-1346 (1997).

11. Bustamante, C., Marko, J. F., Siggia, E. D. \& Smith, S. Entropic elasticity of lambda-phage DNA. Science 265, 1599-1600 (1994).

12. Wuite, G. J., Smith, S. B., Young, M., Keller, D. \& Bustamante, C. Single-molecule studies of the effect of template tension on T7 DNA polymerase activity. Nature 404, 103-106 (2000).

13. Maier, B., Bensimon, D. \& Croquette, V. Replication by a single DNA polymerase of a stretched single-stranded DNA. Proc. Natl Acad. Sci. USA 97, 12002-12007 (2000).

14. Strick, T. R., Allemand, J. F., Bensimon, D., Bensimon, A. \& Croquette, V. The elasticity of a single supercoiled DNA molecule. Science 271, 1835-1837 (1996)

15. Strick, T. R., Croquette, V. \& Bensimon, D. Single-molecule analysis of DNA uncoiling by a type II topoisomerase. Nature 404, 901-904 (2000)

16. Dekker, N. H. et al. The mechanism of type IA topoisomerases. Proc. Natl Acad. Sci. USA 99, 12126-12131 (2002)

17. Crisona, N. J., Strick, T. R., Bensimon, D., Croquette, V. \& Cozzarelli, N. R. Preferential relaxation of positively supercoiled DNA by E. coli topoisomerase IV in single-molecule and ensemble measurements. Genes Dev. 14, 2881-2892 (2000).

18. Essevaz-Roulet, B., Bockelmann, U. \& Heslot, F. Mechanical separation of the complementary strand of DNA. Proc. Natl Acad. Sci. USA 94, 11935-11940 (1997).

19. Bockelmann, U., Thomen, P., Essevaz-Roulet, B., Viasnoff, V. \& Heslot, F. Unzipping DNA with optical tweezers: high sequence sensitivity and force flips. Biophys. J. 82, 1537-1553 (2002)

20. Bianco, P. R. et al. Processive translocation and DNA unwinding by individual RecBCD enzyme molecules. Nature 409, 374-378 (2001).

21.Dohoney, K. M. \& Gelles, J. $\chi$-Sequence recognition and DNA translocation by single RecBCD helicase/nuclease molecules. Nature 409, 370-374 (2001).

22. Koch, S. J., Shundrovsky, A., Jantzen, B. C. \& Wang, M. D. Probing protein-DNA interactions by unzipping a single DNA double helix. Biophys. J. 83, 1098-1105 (2002).

23. Yin, H. et al. Transcription against an applied force. Science 270, 1653-1657 (1995).

24. Wang, M. D. et al. Force and velocity measured for single molecules of RNA polymerase. Science $\mathbf{2 8 2}$ 902-907 (1998).

25. Davenport, R. J., Wuite, G. J., Landick, R. \& Bustamante, C. Single-molecule study of transcriptional pausing and arrest by E. coli RNA polymerase. Science 287, 2497-2500 (2000).

26. Vale, R. D. \& Milligan, R. A. The way things move: looking under the hood of molecular motor proteins. Science 288, 88-95 (2000)

27. Forde, N. R., Izhaky, D., Woodcock, G. R., Wuite, G. J. \& Bustamante, C. Using mechanical force to probe the mechanism of pausing and arrest during continuous elongation by Escherichia coli RNA polymerase. Proc. Natl Acad. Sci. USA 99, 11682-11687 (2002).

28. Landick, R. RNA polymerase slides home: pause and termination site recognition. Cell 88, 741-744 $(1997)$

29. Cui, Y. \& Bustamante, C. Pulling a single chromatin fiber reveals the forces that maintain its higherorder structure. Proc. Natl Acad. Sci. USA 97, 127-132 (2000).

30. Bennink, M. L. et al. Unfolding individual nucleosomes by stretching single chromatin fibers with optical tweezers. Nature Struct. Biol. 8, 606-610 (2001).

31. Brower-Toland, B. D. et al. Mechanical disruption of individual nucleosomes reveals a reversible multistage release of DNA. Proc. Natl Acad. Sci. USA 99, 1960-1965 (2002).

32. Smith, D. E. et al. The bacteriophage $\phi 29$ portal motor can package DNA against a large internal force Nature $413,748-752(2001)$.

33. Calladine, C. R. \& Drew, H. Understanding DNA (Academic, London, 1997).

34. Cluzel, P. et al. DNA: an extensible molecule. Science 271, 792-794 (1996).

35. Leger, J. F. et al. Structural transitions of a twisted and stretched DNA molecule. Phys. Rev. Lett. 83 1066-1069 (1999).

36. Williams, M. C., Rouzina, I. \& Bloomfield, V. A. Thermodynamics of DNA interactions from single molecule stretching experiments. Acc. Chem. Res. 35, 159-166 (2002).

37. Wilkins, M. H. F., Gosling, R. G. \& Seeds, W. E. Nucleic acid: an extensible molecule? Nature 167, 759-760 (1951).

38. Strick, T. R., Allemand, J. F., Bensimon, D. \& Croquette, V. Behavior of supercoiled DNA. Biophys. J. 74, 2016-2028 (1998)

39. Allemand, J. F., Bensimon, D., Lavery, R. \& Croquette, V. Stretched and overwound DNA forms Pauling-like structure with exposed bases. Proc. Natl Acad. Sci. USA 95, 14152-14157 (1998).

40. Pauling, L. \& Corey, R. B. A proposed structure for the nucleic acids. Proc. Natl Acad. Sci. USA 39, 84-97 (1953).

41. Sarkar, A., Leger, J. F., Chatenay, D. \& Marko, J. F. Structural transitions in DNA driven by external force and torque. Phys. Rev. E 63, 051903-1-051903-10 (2001)

42. Harada, Y. et al. Direct observation of DNA rotation during transcription by Escherichia coli RNA polymerase. Nature 409,113-115 (2001).

43. Liphardt, J., Onoa, B., Smith, S. B., Tinoco, I. J. \& Bustamante, C. Reversible unfolding of single RNA molecules by mechanical force. Science 292, 733-737 (2001).

44. Hegner, M. DNA Handles for single molecule experiments. Single Mol. 1, 139-144 (2000).

45. Carrion-Vazquez, M. et al. Mechanical design of proteins studied by single-molecule force spectroscopy and protein engineering. Prog. Biophys. Mol. Biol. 74, 63-91 (2000).

46. Yurke, B., Turberfield, A. J., Mills, A. P., Simmel, F. C. \& Neumann, J. L. A DNA-fuelled molecular machine made of DNA. Nature 406, 605-608 (2000).

47. Yan, H., Zhang, X., Shen, Z. \& Seeman, N. C. A robust DNA mechanical device controlled by hybridization topology. Nature 415, 62-65 (2002).

48. Mao, C., Sun, W., Shen, Z. \& Seeman, N. C. A nanomechanical device based on the B-Z transition of DNA. Nature 397, 144-146 (1999).

49. Strick, T., Allemand, J., Croquette, V. \& Bensimon, D. Twisting and stretching single DNA molecules. Prog. Biophys. Mol. Biol. 74, 115-140 (2000).

50. Alberts, B. et al. Molecular Biology of the Cell (Garland, New York, 2002).
DNA in

a material world

Nadrian C. Seeman

Department of Chemistry, New York University, New York 10003, USA

(e-mail:ned.seeman@nyu.edu)

The specific bonding of DNA base pairs provides the chemical foundation for genetics. This powerful molecular recognition system can be used in nanotechnology to direct the assembly of highly structured materials with specific nanoscale features, as well as in DNA computation to process complex information. The exploitation of DNA for material purposes presents a new chapter in the history of the molecule.

“The nucleic-acid 'system' that operates in terrestrial life is optimized (through evolution) chemistry incarnate. Why not use it ... to allow human beings to sculpt something new, perhaps beautiful, perhaps useful, certainly unnatural." Roald Hoffmann, writing in American Scientist, 1994 (ref. 1).

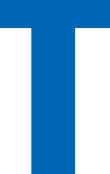

he DNA molecule has appealing features for use in nanotechnology: its minuscule size, with a diameter of about 2 nanometres, its short structural repeat (helical pitch) of about 3.4-3.6 nm, and its 'stiffness', with a persistence length (a measure of stiffness) of around $50 \mathrm{~nm}$. There are two basic types of nanotechnological construction: 'top-down' systems are where microscopic manipulations of small numbers of atoms or molecules fashion elegant patterns (for example, see ref. 2), while in 'bottom-up' constructions, many molecules self-assemble in parallel steps, as a function of their molecular recognition properties. As a chemically based assembly system, DNA will be a key player in bottom-up nanotechnology.

The origins of this approach date to the early 1970s, when in vitro genetic manipulation was first performed by tacking together molecules with 'sticky ends'. A sticky end is a short single-stranded overhang protruding from the end of a double-stranded helical DNA molecule. Like flaps of Velcro, two molecules with complementary sticky ends - that is, their sticky ends have complementary arrangements of the nucleotide bases adenine, cytosine, guanine and thymine - will cohere to form a molecular complex.

Sticky-ended cohesion is arguably the best example of programmable molecular recognition: there is significant diversity to possible sticky ends ( $4^{N}$ for $N$-base sticky ends), and the product formed at the site of this cohesion is the classic DNA double helix. Likewise, the convenience of solid support-based DNA synthesis ${ }^{3}$ makes it is easy to program diverse sequences of sticky ends. Thus, sticky ends offer both predictable control of intermolecular associations and predictable geometry at the point of cohesion. Perhaps one could get similar affinity properties from antibodies and antigens, but, in contrast to DNA sticky ends, the relative three-dimensional orientation of the antibody and the antigen would need to be determined for every new pair. The nucleic acids seem to be unique in this regard, providing a tractable, diverse and programmable system with remarkable control over intermolecular interactions, coupled with known structures for their complexes.

\section{Branched DNA}

There is, however, a catch; the axes of DNA double helices are unbranched lines. Joining DNA molecules by sticky ends can yield longer lines, perhaps with specific components in a particular linear 
Figure 1 Assembly of branched DNA molecules. a, Self-assembly of branched DNA molecules into a two-dimensional crystal. A DNA branched junction forms from four DNA strands; those strands coloured green and blue have complementary sticky-end overhangs labelled $\mathrm{H}$ and $\mathrm{H}^{\prime}$, respectively, whereas those coloured pink and red have complementary overhangs $V$ and $V^{\prime}$, respectively. A number of DNA branched junctions cohere based on the orientation of their complementary sticky ends, forming a square-like unit with unpaired sticky ends on the outside, so more units could be added to produce a two-dimensional crystal. b, Ligated DNA molecules form interconnected rings to create a cube-like structure. The structure consists of six cyclic interlocked single strands, each linked twice to its four neighbours, because each edge contains two turns of the DNA
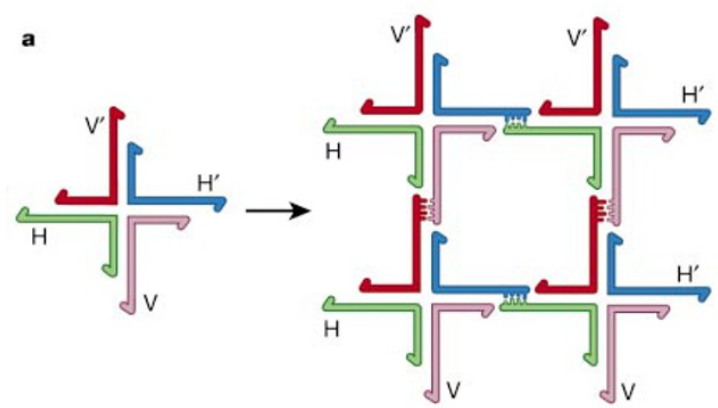

b

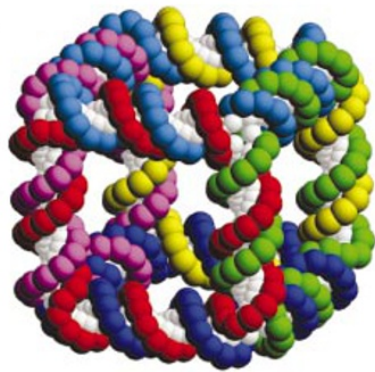

double helix. For example, the front red strand is linked to the green strand on the right, the light blue strand on the top, the magenta strand on the left, and the dark blue strand on the bottom. It is linked only indirectly to the yellow strand at the rear.

or cyclic order in one dimension. Indeed, the chromosomes packed inside cells exist as just such one-dimensional arrays. But to produce interesting materials from DNA, synthesis is required in multiple dimensions and, for this purpose, branched DNA is required.

Branched DNA occurs naturally in living systems, as ephemeral intermediates formed when chromosomes exchange information during meiosis, the type of cell division that generates the sex cells (eggs and sperm). Prior to cell division, homologous chromosomes pair, and the aligned strands of DNA break and literally cross over one another, forming structures called Holliday junctions. This exchange of adjacent sequences by homologous chromosomes - a process called recombination - during the formation of sex cells passes genetic diversity onto the next generation.

The Holliday junction contains four DNA strands (each member of a pair of aligned homologous chromosomes is composed of two DNA strands) bound together to form four double-helical arms flanking a branch point (Fig. 1a). The branch point can relocate throughout the molecule, by virtue of the homologous sequences. In contrast, synthetic DNA complexes can be designed to have fixed branch points containing between three and at least eight $\mathrm{arms}^{4,5}$. Thus, the prescription for using DNA as the basis for complex materials with nanoscale features is simple: take synthetic branched DNA molecules with programmed sticky ends, and get them to self-assemble into the desired structure, which maybe a closed object or a crystalline array (Fig. 1a).

Other modes of nucleic acid interaction aside from sticky ends are available. For example, Tecto-RNA molecules ${ }^{6}$, held together by loop-loop interactions, or paranemic crossover (PX) DNA, where cohesion derives from pairing of alternate half turns in inter-wrapped double helices ${ }^{7}$. These new binding modes represent programmable cohesive interactions between cyclic single-stranded molecules that do not require cleavage to expose bases to pair molecules together. Nevertheless, cohesion using sticky ends remains the most prominent intermolecular interaction in structural DNA nanotechnology.

\section{DNA constructions}

It is over a decade since the construction of the first artificial DNA structure, a stick-cube, whose edges are double helices ${ }^{8}$ (Fig. 1b). More complex polyhedra and topological constructs ${ }^{9}$, such as knots and Borromean rings (consisting of three intricately interlinked circles), followed. But the apparent floppiness of individual branched junctions led to a hiatus before the next logical step: selfassembly into two-dimensional arrays.

This step required a stiffer motif, as it was difficult to build a periodic well-structured array with marshmallow-like components,

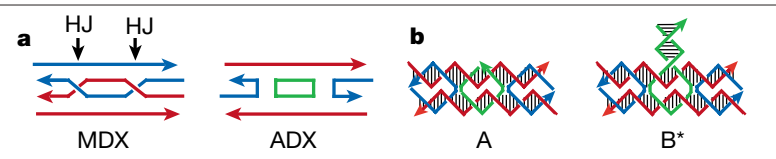
crossover (DX) units. In the meiotic DX recombination intermediate, labelled MDX, a pair of homologous chromosomes, each consisting of two DNA strands, align and cross over in order to swap equivalent portions of genetic information; ' $\mathrm{HJ}$ ' indicates the Holliday junctions. The structure of an analogue unit ( $\mathrm{ADX}$ ), used as a tiling unit in the construction of DNA two-dimensional arrays, comprises two red strands, two blue crossover strands and a central green crossover strand. $\mathbf{b}$. The strand structure and base pairing of the analogue ADX molecule, labelled $A$, and a variant, labelled $B^{*}$. $B^{*}$ contains an extra DNA domain extending from the central green strand that, in practice, protrudes roughly perpendicular to the plane of the rest of the DX molecule. c, Schematic representations of $A$ and $B^{*}$ where the perpendicular domain of $B^{*}$ is represented as a blue circle. The complementary ends of the ADX molecules are represented as geometrical shapes to illustrate how they fit together when they selfassemble. The dimensions of the resulting tiles are about $4 \times 16 \mathrm{~nm}$ and are joined together so that the $B^{*}$ protrusions lie about $32 \mathrm{~nm}$ apart. $\mathbf{d}$, The $B^{*}$ protrusions are visible as 'stripes' in tiled DNA arrays under an atomic force microscope.

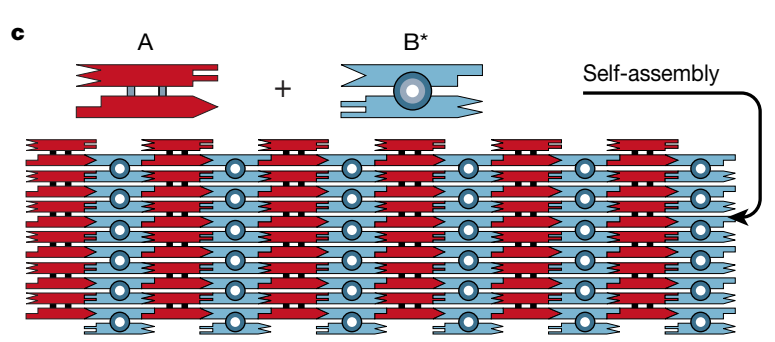

d

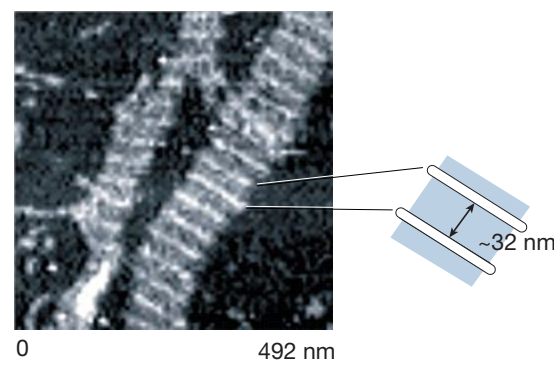




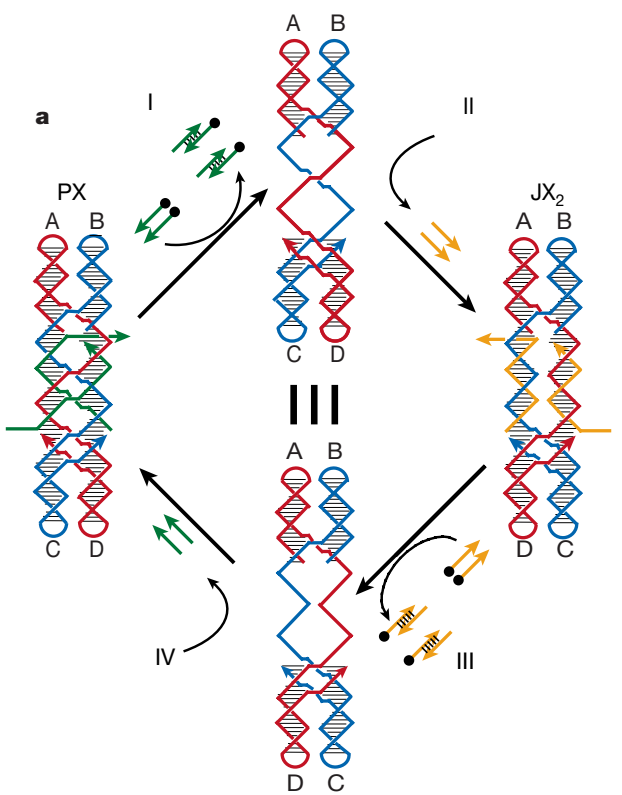

Figure 3 A rotary DNA nanomachine. a, The device works by producing two different conformations, depending on which of two pairs of strands (called 'set' strands) binds to the device framework. The device framework consists of two DNA strands (red and blue) whose top and bottom double helices are each connected by single strands. Thus, they form two rigid arms with a flexible hinge in between and the loose ends of the two strands dangling freely. The two states of the device, $\mathrm{PX}$ (left) and $\mathrm{JX}_{2}$ (right), differ by a half turn in the relative orientations of their bottom helices ( $C$ and $D$ on the left, $D$ and $C$ on the right). The difference between the two states is analogous to two adjacent fingers extended, parallel to each other (right), or crossed (left). The states are set by the presence of green or yellow set strands, which bind to the frame in different ways to produce different conformations. The set strands have extensions that enable their removal when complementary strands are added (steps I and III). When one type of set strand is removed, the device is free to bind the other set strands and switch to a different state (steps II and IV). b. The PX-JX device can be used to connect 20-nm DNA trapezoid constructs. In the PX state, they are in a parallel conformation, but in the $\mathrm{JX}_{2}$ state, they are in a zig-zag conformation, which can be visualized on the right by atomic force microscopy. even with a well-defined blueprint (sticky-ended specificity) for their assembly. The stiffer motif was provided by the DNA doublecrossover (DX) molecule ${ }^{10}$, analogous, once again, to the double Holliday-junction intermediate formed during meiosis (MDX, Fig. 2a). This stiff molecule contains two double helices connected to each other twice through crossover points. It is possible to program DX molecules to produce a variety of patterned two-dimensional arrays just by controlling their sticky ends ${ }^{11-13}$ (Fig. 2b).

\section{DNA nanomachines}

In addition to objects and arrays, a number of DNA-based nanomechanical devices have been made. The first device consisted of two DX molecules connected by a shaft with a special sequence that could be converted from normal right-handed DNA (known as B-DNA) to an unusual left-handed conformation, known as Z-DNA ${ }^{14}$. The two DX molecules lie on one side of the shaft before conversion and on opposite sides after conversion, which leads to a rotation. The problem with this device is that it is activated by a small molecule, $\mathrm{Co}\left(\mathrm{NH}_{3}\right)_{6}^{3+}$, and with all devices sharing the same stimulus, an ordered collection of DX molecules would not produce a diversity of responses.

This problem was solved by Bernard Yurke and colleagues, who developed a protocol for a sequence-control device that has a tweezers-like motion ${ }^{15}$. The principle behind the device is that a so-called 'set' strand containing a non-pairing extension hybridizes to a DNApaired structural framework and sets a conformation; another strand that is complementary to the 'set' strand is then added, which binds to both the pairing and non-pairing portions, and removes it from the structure, leaving only the framework.

A robust rotary device was developed based on this principle ${ }^{16}$ (Fig. 3), in which different set strands can enter and set the conformation to different structural end-states. In this way, the conformation of the DNA device can readily be flipped back and forth simply by adding different set strands followed by their complements. A variety of different devices can be controlled by a diverse group of set strands.
Figure 4 Applications of DNA scaffolds. a, Scaffolding of biological macromolecules. a A DNA box (red) is shown with protruding sticky ends that are used to organize boxes into crystals. Macromolecules are organized parallel to each other within the box, rendering them amenable to structure determination by $\mathrm{X}$-ray crystallography. b, DNA scaffolds to direct the assembly of nanoscale electrical circuits. Branched DNA junctions (blue) direct the assembly of attached nanoelectronic components (red), which are stabilized by the addition of a positively charged ion.
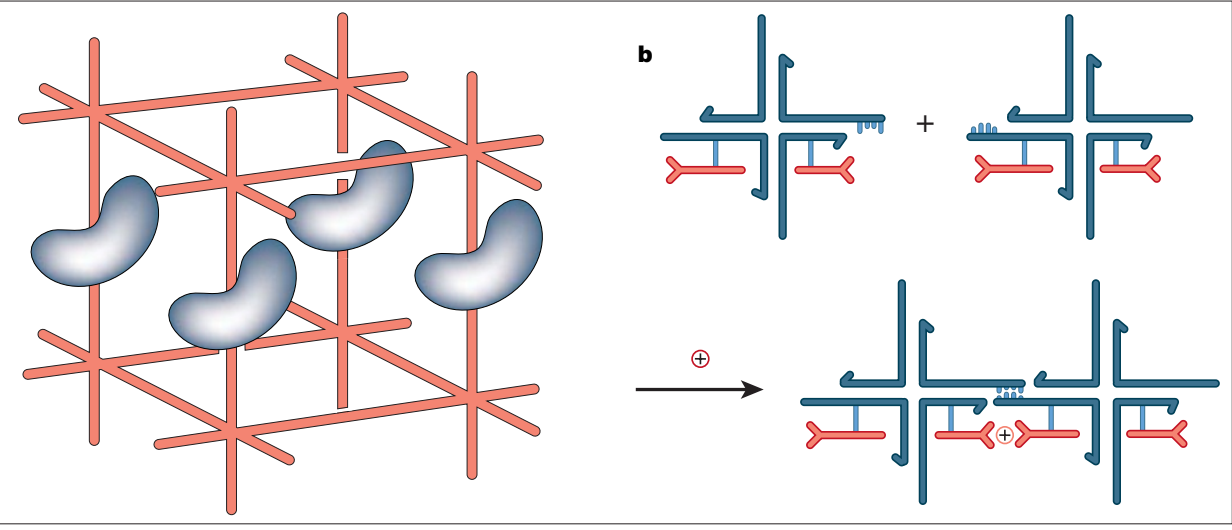


\section{DNA as a scaffold}

What is the purpose of constructing DNA arrays and nanodevices? One prominent goal is to use DNA as scaffolding to organize other molecules. For example, it may be possible to use self-assembled DNA lattices (crystals) as platforms to position biological macromolecules so as to study their structure by X-ray crystallography ${ }^{4}$ (Fig. 4a). Towards this goal, programming of DNA has been used to bring protein molecules in proximity with each other to fuse multiple enzymatic activities ${ }^{17}$. However, the potential of this approach awaits the successful self-assembly of three-dimensional crystals.

Another goal is to use DNA crystals to assemble nanoelectronic components in two- or three-dimensional arrays ${ }^{18}$ (Fig. 4b). DNA has been shown to organize metallic nanoparticles as a precursor to nanoelectronic assembly ${ }^{19-22}$, but so far it has not been possible to produce multidimensional arrays containing nanoelectronic components with the high-structural order of the naked DNA arrays described earlier.

There has been some controversy over whether DNA can be used as an electrical conductor (for example, ref. 23), although the resolution of this debate is unlikely have any impact on the use of DNA as a scaffold. Recently, the effects of DNA conformational changes on conduction in the presence of an analyte were shown to have potential as a biosensor ${ }^{24}$.

\section{Replicating DNA components}

A natural question to ask of any assembly system based on DNA is whether the components can be replicated. To produce branched DNA molecules whose branch points do not move, they must have different sequences in opposite branches but, as a consequence, these structures are not readily reproduced by DNA polymerase; the polymerase would produce complements to all strands present, leading only to double helical molecules. Oneoption is to use topological tricks to convert structures like the DNA cubeinto a long single strand by adding extra stretches of DNA bases. The single strand could then be replicated by DNA polymerase and the final replicated product induced to fold into the original shape, with any extraneous segments cleaved using restriction enzymes. Although this would produce a molecule with sticky ends ready to participate in self-assembly, it would be a cumbersome process $\mathrm{s}^{25}$.

Günter von Kiedrowski and colleagues have recently developed a way of replicating short, simple DNA branches in a mixed organic-DNA species. Their branched molecule consists of three DNA single strands bonded to an organic triangle-shaped linker. To replicate the branched molecule, the single-stranded complement of each of these strands is bound to the molecule, so that one end of each complement molecule is close to the same end of the other complement molecule. In the final step, the juxtaposed complements are connected together by bonding their neighbouring ends to another molecule of the organic linker ${ }^{26}$. Extension of this system to the next level, such as objects like the cube, will need to solve topological problems involved in the separation of the two components, or it will belimited to unligated systems.

\section{Future prospects}

Many separate capabilities of DNA nanotechnology have been prototyped - it is now time to extend and integrate them into useful systems. Combining sequence-dependent devices with nanoscale arrays will provide a system with a vast number of distinct, programmable structural states, the sine qua non of nanorobotics. A key step in realizing these goals is to achieve highly ordered three-dimensional arrays, both periodic and, ultimately, algorithmic.

Interfacing with top-down nanotechnology will extend markedly the capabilities of the field. It also will be necessary to integrate biological macromolecules or other macromolecular complexes into DNA arrays in order to make practical systems with nanoscale components. Likewise, the inclusion of electronic components in highly ordered arrays will enable the organization of nanoelectronic circuits. Chemical function could be added to DNA arrays by adding nucleic acid species evolved in vitro to have specific binding properties ('aptamers') or enzymatic activities ('ribozymes' or 'DNAzymes'). A further area that has yet to have an impact on DNA nanotechnology is

Box 1

DNA computers

An assembly of DNA strands can process data in a similar way as an electronic computer, and has the potential to solve far more complex problems and store a greater amount of information, for substantially less energy costs than do electronic microprocessors. DNA-based computation dates from Leonard Adleman's landmark report in 1994 (ref. 27), where he used DNA to solve the 'Hamiltonian path' problem, a variant of the 'travelling salesman' problem. The idea is to establish whether there is a path between two cities, given an incomplete set of available roads. Adleman used strands of DNA to represent cities and roads, and encoded the sequences so that a strand representing a road would connect (according to the rules of base pairing) to any two strands representing a city. By mixing together the strands, joining the cities connected by roads, and weeding out any 'wrong answers', he showed that the strands could self-assemble to solve the problem.

It is impossible to separate DNA nanotechnology from DNAbased computation: many researchers work in both fields and the two communities have a symbiotic relationship. The first link between DNA computation and DNA nanotechnology was established by Erik Winfree, who suggested that short branched DNA molecules could be 'programmed' to undergo algorithmic selfassembly and thus serve as the basis of computation ${ }^{28}$.

Periodic building blocks of matter, such as the DNA molecules shown in Fig. 1a, represent the simplest algorithm for assembly. All components are parallel, so what is on one side of a component is also on the other side, and in every direction. Given this parallelism, if the right side complements the left, the top complements the bottom and the front complements the back, a crystal should result. Even more complex algorithms are possible if one uses components of the same shape, but with different sticky ends. For example, Winfree has shown that, in principle, DNA tiles can be used to 'count' (see figure below) by creating borders with programmable sizes for one-, two- and possibly three-dimensional assemblies ${ }^{29}$. If this scheme can be realized, self-assembly of precisely sized nanoscale arrays will be possible. A computation using selfassembly has been prototyped in one dimension, thereby lending some credence to the viability of algorithmic assembly ${ }^{30}$.

Box 1 Figure Counting with self-assembled DNA tiles. DNA tiles are represented by squares with coloured edges that are protruded or indented. Seven component tiles are shown on the left: three border tiles on the bottom and four tiles with the values $\mathbf{0}$ or $\mathbf{1}$. The array illustrates binary counting from 1 (bottom row) to 12 (top row). Assembly is assumed to proceed by forming the reverse $L$-shaped border first, followed by binding the tiles that fit into the sites containing two (but not one) edges. Thus, the border determines the $\mathbf{1}$ tile in its bend, then that 1 tile and the horizontal-border tile on its left determine the $\mathbf{0}$ tile that fits, while the $\mathbf{1}$ tile and the vertical-border tile above it determine the (different) $\mathbf{0}$ tile that fits. (Adapted from ref. 29.) 
combinatorial synthesis, which may well lead to greater diversity of integrated components. DNA-based computation and algorithmic assembly is another active area of research, and one that is impossible to separate from DNA nanotechnology (see Box 1).

The field of DNA nanotechnology has attracted an influx of researchers over the past few years. All of those involved in this area have benefited from the biotechnology enterprise that produces DNAmodifying enzymes and unusual components for synthetic DNA molecules. It is likely that applications in structural DNA nanotechnology ultimately will use variants on the theme of DNA (for example, peptide nucleic acids, containing an unconventional synthetic peptide backbone and nucleic acid bases for side chains), whose properties may be better suited to particular types of applications.

For the past half-century, DNA has been almost exclusively the province of biologists and biologically oriented physical scientists, who have studied its biological impact and molecular properties. During the next 50 years, it is likely they will be joined by materials scientists, nanotechnologists and computer engineers, who will exploit DNA's chemical properties in a non-biological context.

doi:10.1038/nature01406

1. Hoffmann, R. DNA as clay. Am. Sci. 82, 308-311 (1994).

2. Cuberes, M. T., Schlittler, R. R. \& Gimzewski, J. K. Room-temperature repositioning of individual C-60 molecules at Cu steps: operation of a molecular counting device. Appl. Phys. Lett. 69, 3016-3018 (1996).

3. Caruthers, M. H. Gene synthesis machines: DNA chemistry and its uses. Science 230, 281-285 (1985).

4. Seeman, N. C. Nucleic acid junctions and lattices. J. Theor. Biol. 99, 237-247 (1982)

5. Seeman, N. C. Molecular craftwork with DNA. Chem. Intell. 1, 38-47 (1995).

6. Jaeger, L., Westhof, E. \& Leontis, N. B. Tecto-RNA: modular assembly units for the construction of RNA nano-objects. Nucleic Acids Res. 29, 455-463 (2001).

7. Zhang, X., Yan, H., Shen, Z. \& Seeman, N. C. Paranemic cohesion of topologically-closed DNA molecules. J. Am. Chem. Soc. 124, 12940-12941 (2002).

8. Chen, J. \& Seeman, N. C. The synthesis from DNA of a molecule with the connectivity of a cube. Nature 350, 631-633 (1991).

9. Seeman, N. C. Nucleic acid nanostructures and topology. Angew. Chem. Int. Edn Engl. 37, 3220-3238 (1998)

10.Li, X., Yang, X., Qi, J. \& Seeman, N. C. Antiparallel DNA double crossover molecules as components for nanoconstruction. J. Am. Chem. Soc. 118, 6131-6140 (1996).

11. Winfree, E., Liu, F., Wenzler, L.A. \& Seeman, N.C. Design and self-assembly of two-dimensional DNA crystals. Nature 394, 539-544 (1998).

12. Mao, C., Sun, W. \& Seeman, N. C. Designed two-dimensional DNA Holliday junction arrays visualized by atomic force microscopy. J. Am. Chem. Soc. 121, 5437-5443 (1999).

13. LaBean, T. et al. The construction, analysis, ligation and self-assembly of DNA triple crossover complexes. J. Am. Chem. Soc. 122, 1848-1860 (2000).

14. Mao, C., Sun, W., Shen, Z. \& Seeman, N. C. A DNA nanomechanical device based on the B-Z transition. Nature 397, 144-146 (1999).

15. Yurke, B., Turberfield, A. J., Mills, A. P. Jr, Simmel, F. C. \& Newmann, J. L. A DNA-fuelled molecular machine made of DNA. Nature 406, 605-608 (2000).

16. Yan, H., Zhang, X., Shen, Z. \& Seeman, N. C. A robust DNA mechanical device controlled by hybridization topology. Nature 415, 62-65 (2002).

17. Niemeyer, C. M., Koehler, J. \& Wuerdemann, C. DNA-directed assembly of bi-enzymic complexes from in vivo biotinylated $\mathrm{NADP}(\mathrm{H})$ : $\mathrm{FMN}$ oxidoreductase and luciferase. ChemBioChem 3, 242-245 (2002).

18. Robinson, B. H. \& Seeman, N. C. The design of a biochip: a self-assembling molecular-scale memory device. Protein Eng. 1, 295-300 (1987).

19. Keren, K. et al. Sequence-specific molecular lithography on single DNA molecules. Science 297, 72-75 (2002).

20. Alivisatos, A. P. et al. Organization of 'nanocrystal molecules' using DNA. Nature 382, 609-611 (1996).

21. Taton, T. A., Mucic, R. C., Mirkin, C. A. \& Letsinger, R. L. The DNA-mediated formation of supramolecular mono- and multilayered nanoparticle structures. J. Am. Chem. Soc. 122, 6305-6306 (2000).

22. Pena, S. R. N., Raina, S., Goodrich, G. P., Fedoroff, N. V. \& Keating, C. D. Hybridization and enzymatic extension of Au nanoparticle-bound oligonucleotides. J. Am. Chem. Soc. 124, 7314-7323 (2002).

23. Dekker, C. \& Ratner, M. A. Electronic properties of DNA. Phys. World 14, 29-33 (2001).

24. Fahlman, R. P. \& Sen, D. DNA conformational switches as sensitive electronic sensors of analytes. J. Am. Chem. Soc. 124, 4610-4616 (2002).

25. Seeman, N. C. The construction of 3-D stick figures from branched DNA. DNA Cell Biol. 10, 475-486 (1991).

26. Eckardt, L. H. et al. Chemical copying of connectivity. Nature 420, 286 (2002).

27. Adleman, L. Molecular computation of solutions to combinatorial problems. Science 266, 1021-1024 (1994)

28. Winfree, E. in DNA Based Computers. Proceedings of a DIMACS Workshop, April 4, 1995, Princeton University (eds Lipton, R. J \& Baum, E. B.) 199-219 (American Mathematical Society, Providence, 1996).

29. Winfree, E. Algorithmic self-assembly of DNA: theoretical motivations and 2D assembly experiments. J. Biol. Mol. Struct. Dynamics Conversat. 11 2, 263-270 (2000).

30. Mao, C., LaBean, T., Reif, J. H. \& Seeman, N. C. Logical computation using algorithmic self-assembly of DNA triple crossover molecules. Nature 407, 493-496 (2000).

\section{Acknowledgements}

This work has been supported by grants from the National Institute of General Medical Sciences, the Office of Naval Research, the National Science Foundation, and the Defense Advanced Research Projects Agency/Air Force Office of Scientific Research.

\section{DNA replication and recombination}

\section{Bruce Alberts}

National Academy of Sciences, 2101 Constitution Avenue,

Washington DC 20418, USA

Knowledge of the structure of DNA enabled scientists to undertake the difficult task of deciphering the detailed molecular mechanisms of two dynamic processes that are central to life: the copying of the genetic information by DNA replication, and its reassortment and repair by DNA recombination. Despite dramatic advances towards this goal over the past five decades, many challenges remain for the next generation of molecular biologists.

"Though facts are inherently less satisfying than the intellectual conclusions drawn from them, their importance should never be questioned." James D. Watson, 2002.

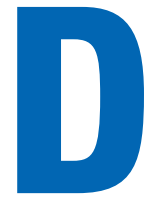

NA carries all of the genetic information for life. One enormously long DNA molecule forms each of the chromosomes of an organism, 23 of them in a human. The fundamental living unit is the single cell. A cell gives rise to many more cells through serial repetitions of a process known as cell division. Before each division, new copies must be made of each of the many molecules that form the cell, including the duplication of all DNA molecules. DNA replication is the name given to this duplication process, which enables an organism's genetic information - its genes - to be passed to the two daughter cells created when a cell divides. Only slightly less central to life is a process that requires dynamic DNA acrobatics, called homologous DNA recombination, which reshuffles the genes on chromosomes. In reactions closely linked to DNA replication, the recombination machinery also repairs damage that inevitably occurs to the long, fragile DNA molecules inside cells (see article in this issue by Friedberg, page 436).

The model for the DNA double helix ${ }^{1}$ proposed by James Watson and Francis Crick is based on two paired DNA strands that are complementary in their nucleotide sequence. The model had striking implications for the processes ofDNA replication and DNA recombination. Before 1953, there had been no meaningful way of even speculating about the molecular mechanisms of these two central genetic processes. But the proposal that each nucleotide in one strand of DNA was tightly base-paired with its complementary nucleotide on the opposite strand - either adenine $(A)$ with thymine $(T)$, or guanine $(G)$ with cytosine $(\mathrm{C})$ - meant that any part of the nucleotide sequence could act as a direct template for the corresponding portion of the other strand. As a result, any part of the sequence can be used either to create or to recognize its partner nucleotide sequence - the two functions that are central for DNA replication and DNA recombination, respectively.

In this review, I discuss how the discovery of the structure of DNA half a century ago opened new avenues for understanding the processes of DNA replication and recombination. I shall also emphasize how, as our understanding of complex biological molecules and their interactions increased over the years, there have been profound changes in the way that biologists view the chemistry of life.

\section{Structural features of DNA}

The research that immediately followed the discovery of the double helix focused primarily on understanding the structural properties 